\title{
Self-localization method for mobile robot using acoustic beacons
}

\author{
Satoki Ogiso ${ }^{1}$, Takuji Kawagishi ${ }^{2}$, Koichi Mizutani ${ }^{3 *}$, Naoto Wakatsuki ${ }^{3}$ and Keiichi Zempo ${ }^{3}$
}

\begin{abstract}
In this paper, we have proposed a low-cost self-localization method which uses 4 elements of microphones, wheel rotation and sound sources as beacons, whose absolute location and frequency bands are known. The proposed method consists of following 4 steps. The proposed method (i) execute self-localization using wheel-based odometry, (ii) estimate direction-of-arrival (DOA) of the sound sources using sounds recorded by the elements of the microphone array, (iii) predict the DOA of the sound sources from estimated location and pose, and (iv) conduct self-localization by integrating all of the information. To evaluate the proposed method, experiments were conducted. The proposed method was compared to the conventional methods, which were wheel-based odometry and self-localization using only DOA. In the experiments, we have supposed the house-cleaning robot and its trajectory. As results, without any obstacles or walls, the mean of the estimation errors by wheel-based odometry were $670 \mathrm{~mm}$ and $0.08 \mathrm{rad}$, and those of self-localization using only DOA were $2870 \mathrm{~m}$ and $0.07 \mathrm{rad}$ in the worst case. In contrast with these methods, proposed method results in $69 \mathrm{~mm}, 0.02 \mathrm{rad}$ as the worst estimation error of self location and pose. From the result with occlusion of a sound source, the mean of the localization error increased $60 \mathrm{~mm}$, as the proposed method detects the incorrect DOA and prevents it from estimation. From the result with reflective wave from wall, there was a place where the localization error was large. The cause of this error was considered as directivity of sound source. These results indicate that the proposed method is feasible under indoor environment.
\end{abstract}

Keywords: Self-localization; Mobile robot; Acoustic beacons; Microphone array

\section{Background}

Mobile robots are widely used indoor for building security, cleaning, automatic guided vehicle system and so on. For autonomous robots, self-localization is one of the essential function to achieve tasks autonomously. While there are needs for inexpensive robots without precise self-localization, they are generally expensive because of the excessively accurate sensing. To make indoor autonomous robots inexpensive, it is essential to develop the self-localization method which does not require expensive sensor or processor and have just enough accuracy. Conventional self-localization methods are divided mainly into two approaches: the methods which use internal information and the methods which use external information of the robot. Internal information of the robot is measured by internal sensors such as rotary encoders

\footnotetext{
*Correspondence: mizutani@iit.tsukuba.ac.jp

${ }^{3}$ Faculty of Engineering, Information and Systems, University of Tsukuba, Ibaraki, JP
}

Full list of author information is available at the end of the article or accelerometers equipped on the robot [1]. The selflocalization using these internal sensors requires relatively low-calculation cost, as the self-localization using these sensors are merely the accumulation of the information, such as wheel rotation obtained from rotary encoders or accelerations measured by accelerometer. Moreover, the internal sensors and the processor used for self-localization are generally inexpensive. However, once the error, such as bias of the measurement or slip of the robot, was accumulated, it cannot be detected and the estimation error piles up. Sometimes the error would be fatal as the robot cannot reach to the destination or crashes into a facility. While internal sensors are low-cost, external information are usually measured by laser range finder [2], camera [3], ultrasonic range sensor [4] and microphones [5]. With the external information from laser range finder or camera, simultaneous localization and mapping (SLAM) algorithm provides robust self-localization results as it integrates the external

\section{Springer}

(c) Ogiso et al. 2015. Open Access This article is distributed under the terms of the Creative Commons Attribution 4.0 International License (http://creativecommons.org/licenses/by/4.0/), which permits unrestricted use, distribution, and reproduction in any medium, provided you give appropriate credit to the original author(s) and the source, provide a link to the Creative Commons license, and indicate if changes were made. 
information of the robot [6]. However, these sensors are generally expensive and there are much more information to be processed than the internal sensors so that it is hard to be implemented on a low-cost processors, such as microcontrollers. Even if the sensor itself was inexpensive, the extraction of the feature from the obtained data such as image is costly calculation or otherwise the localization need to be achieved by monte-carlo method which requires much memory and calculation cost than simple Kalman filtering [7]. Because of these reasons, most of the self-localization methods with external information require high investment for sensors and computers. However, suitable self-localization method which does not require expensive sensor or processor and does not accumulate the error over time have not been realized.

From the reasons of the sensor cost, there are some researches focused on the sound signals as beacons to self-locate the mobile robot. Previously, many researches have been done for the use of sound signals for robots and several techniques such as the self-localization, sound sources separation and autonomous speech recognition are reported [8-13]. Also there are self-localization methods using microphones installed around the room [14-17]. However, most of them use a huge amount of microphones for self-localization or separation of sound signals to improve accuracy or to suppress the effect of reflective sounds. While these techniques use many microphones, it is difficult to achieve self-localization with only a few number of microphones [5]. We have proposed a self-localization method using only a few number of microphones and conducted simulation to examine it $[18,19]$. This is based on the techniques using microphone array consists of small element number [20-22].

In this paper, we propose a low-cost self-localization method which uses 4 elements of microphones, wheel rotation and known sound sources as beacons. Comparison of conventional low-cost self-localization methods and proposed method is shown in Fig. 1. Wheel-based odometry is one of the most popular self-localization methods, as it is easy to be implemented. However, it is known that the errors of measurements are accumulated and the total estimation error increase over time. In contrast, acoustic localization method does not accumulate errors of measurements, while the estimation results of this method sometimes diverge. The proposed method is the combination of these two. The features of the proposed method are followings:

- The proposed method uses only low cost sensors: a few microphones and rotary encoders.

- The proposed method combines 2 low-cost methods, which have complementary properties, to improve accuracy of each other.

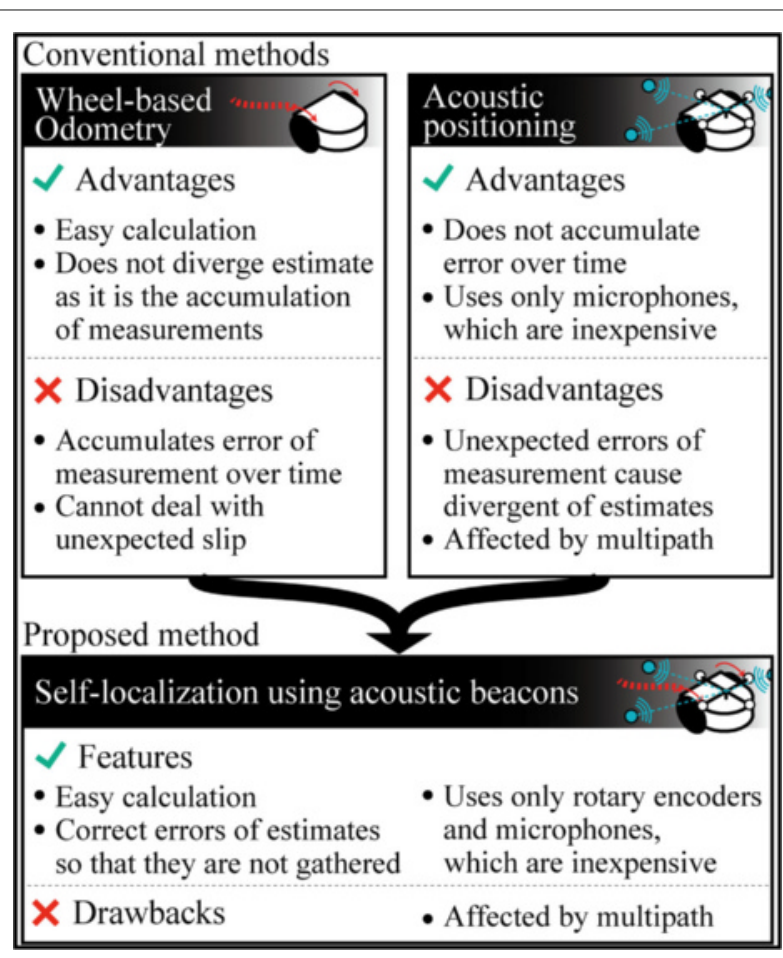

Fig. 1 Comparison of conventional methods and proposed method

- The extraction of the landmark can be easily conducted (e.g. using band-pass filter) relative to the camera image or laser scanned data.

- Extended Kalman filter is used so that it is able to deal with errors of measurements and able to be implemented on a powerless computers.

As this method uses only sound signals and it is not a SLAM problem, the proposed method have the characteristics that the sensors are inexpensive and the calculation cost are relatively low. To evaluate the proposed method, experiment were conducted. The proposed method was compared with the conventional methods, which were wheel-based odometry and self-localization using only DOA.

\section{Methods}

\section{Overview of the proposed method}

The proposed method achieves self-localization by integrating the information from wheel rotation and sound direction-of-arrival(DOA) using extended Kalman filter. DOA is estimated by microphone array which has only 4 elements. The overview of the proposed method is shown in Fig. 2. The proposed method consists of following 4 steps. The proposed method (i) execute wheel-based odometry, (ii) estimate DOA of the beacon sound using sounds recorded by the elements of the microphone array, 


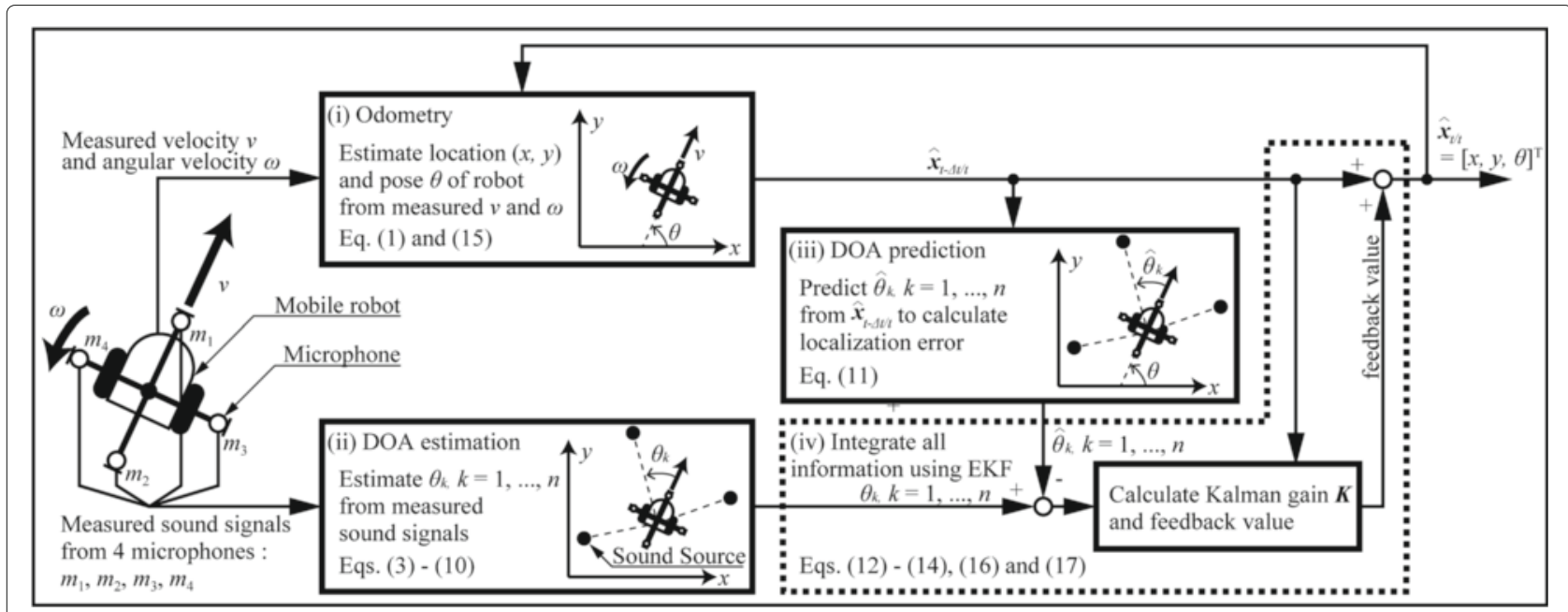

Fig. 2 Overview of the proposed method

(iii) predict the DOA of the beacon sound from estimated location and pose, and (iv) conduct self-localization by integrating all of the information. These steps are described in more detail in the following subsections.

\section{(i) Wheel-based odometry}

In this subsection, wheel-based odometry is described. Coordinate system used in this paper is shown in Fig. 3. Let denote the state of the robot as $\boldsymbol{x}=\left[\begin{array}{lll}x & y & \theta\end{array}\right]^{\mathrm{T}}$ and the time evolution of this state is,

$$
\boldsymbol{f}(\boldsymbol{x})=\boldsymbol{x}+\left[\begin{array}{c}
v \cos \theta \\
v \sin \theta \\
\omega
\end{array}\right] \Delta t
$$

where $v$ and $\omega$ are measured robot velocity and angular velocities, which are measured from wheel rotation. The state of robot in time $t$ could be calculate by the integration of the above formula.

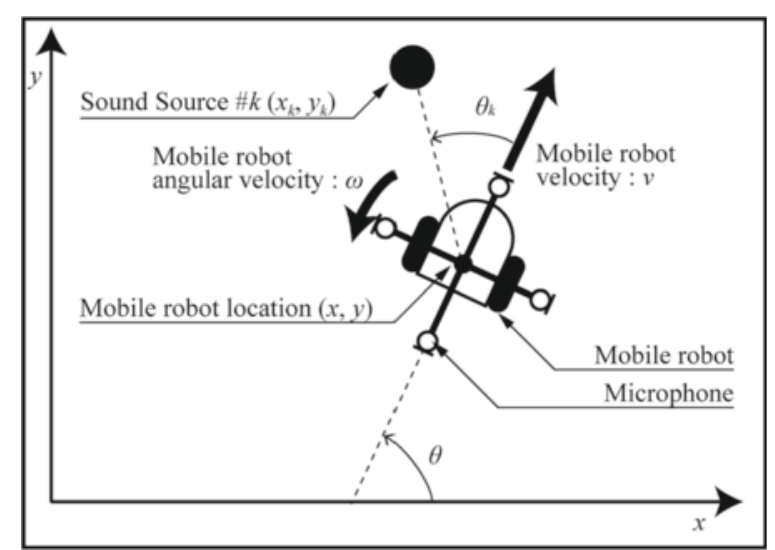

Fig. 3 Coordinate system used in this paper

\section{(ii) Estimation of direction-of-arrival using microphone array}

The angle between the direction of sound source $k$ and the heading of the mobile robot is represented by $\theta_{k}$, as shown in Fig. 4. $\theta_{k}$ is called direction-of-arrival (DOA). We are going to estimate $\theta_{k}$ using signals recorded by microphone array. To estimate $\theta_{k}$, we utilize the relation between $\theta_{k}$ and propagation time differences of sound between the elements of microphone array. Propagation time differences of sound between the elements are measured by cross-correlation method. At first, we describe the cross-correlation method.

Assuming that sound signals propagated from the sound source $\mathrm{k}$ would delay $\tau_{i, k}$ and $\tau_{j, k}$, the received signal on microphone elements $i$ and $j, m_{i, k}$ and $m_{j, k}$, would be,

$$
\begin{gathered}
m_{i, k}(t)=s_{k}\left(t+\tau_{i, k}\right), \\
m_{j, k}(t)=s_{k}\left(t+\tau_{j, k}\right),
\end{gathered}
$$

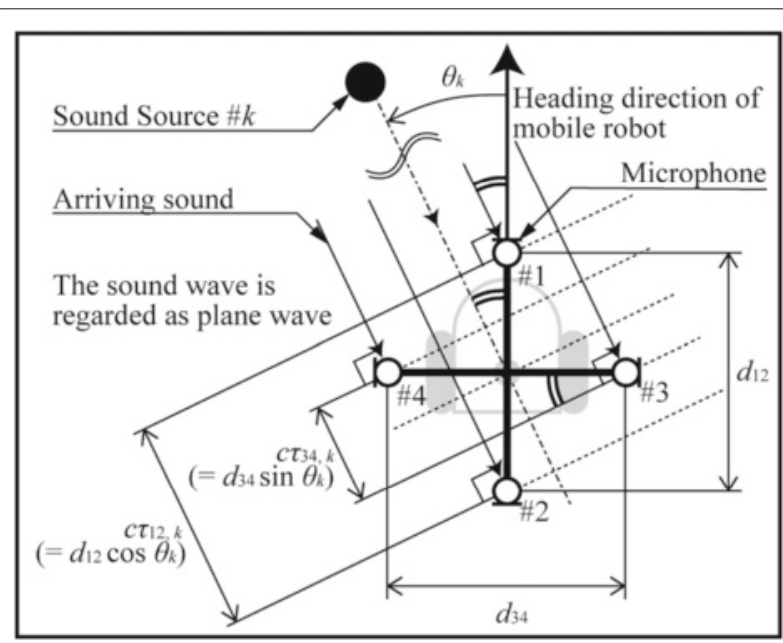

Fig. 4 Direction-of-arrival estimation 
where $s_{k}(t)$ represents the sound signal of the sound source $k$ at time $t$. With these signals and given window length $w$, cross-correlation function $f_{i j, k}(t)$ is calculated as,

$$
f_{i j, k}(t)=\int_{t-w}^{t} m_{i, k}(\tau) m_{j, k}(\tau-t) d \tau .
$$

The peak of $f_{i j, k}(t)$ is at the time when $m_{i, k}$ and $m_{j, k}$ have maximum number of correlation, namely at the time $\tau_{i j, k} \equiv\left|\tau_{i, k}-\tau_{j, k}\right|$. Using this nature of cross-correlation, we can obtain the propagation time difference of the sound from sound source $k$ between the elements $i$ and $j$ as,

$$
\tau_{i j, k}=\arg _{t} \max \left(f_{i j, k}(t)\right) .
$$

Second, we describe the detail of DOA estimation using the relation between propagation time differences and $\theta_{k}$. Assume that we are going to use a microphone array which is shown in Fig. 4. The robot has 4 microphones for the following reason. The microphone array with 2 microphones is the minimum equipment to measure the DOA in two-dimension, however it cannot estimate DOA uniquely as it cannot distinguish whether the sounds come from front or back. The microphone array with 3 microphones is sufficient for unique DOA estimation, however it requires 3 combinations of $\tau_{i j, k}$ to achieve spatially symmetrical estimation of DOA. The microphone array shown in Fig. 4 can estimate DOA uniquely and only 2 combinations $\left(\tau_{12, k}\right.$ and $\left.\tau_{34, k}\right)$ are required for symmetrical estimation DOA.

If the microphone array and sound source $k$ are enough distant from each other, the sound wave from sound source $k$ to the microphone array can be regarded as a plane wave. This wave reaches to the microphone array at an angle of $\theta_{k}$. This angle causes the propagation time difference for each element of microphone array. Measuring this propagation time difference, we can estimate $\theta_{k}$.

From the relation of the time difference of arrival, $\tau_{12, k}$, and distance between microphones, $d_{12}$, the DOA of sound source $k, \theta_{k}$, would be given by solving,

$$
c \tau_{12, k}=d_{12} \sin \theta_{k},
$$

where $c$ represents the sound velocity.

Similarly, this relation is applied to the elements 3 and 4. Let us denote the propagation time difference of the elements 3 and 4 from sound source $k$ by $\tau_{34, k}$, and the distance between the elements 3 and 4 by $d_{34}$. The following equation is derived in a similar way.

$$
c \tau_{34, k}=d_{34} \cos \theta_{k} .
$$

By solving equations (6) and (7) for $\theta_{k}$, the following equation is obtained,

$$
\theta_{k}=\operatorname{atan} 2\left(\frac{\tau_{12, k}}{d_{12}}, \frac{\tau_{34, k}}{d_{34}}\right) .
$$

Here $\operatorname{atan} 2(x, y)$ represents the function which returns the angle of the position $(x, y)$ from $x$-axis in the range of $\left[\begin{array}{ll}-\pi & \pi\end{array}\right]$. Using this equation, $\theta_{k}$ can be estimated.

In practical use, it is known that the DOA estimation can be inaccurate by several reasons such as multi-path. To examine the accuracy of the DOA estimation, the proposed method use the following value $\Delta \tau_{k}$.

$$
\Delta \tau_{k} \equiv 1-\sqrt{\left(\frac{c \tau_{12, k}}{d_{12}}\right)^{2}+\left(\frac{c \tau_{34, k}}{d_{34}}\right)^{2}} .
$$

If the propagation time differences are correctly estimated, $\Delta \tau_{k}$ becomes 0 . This value $\Delta \tau_{k}$ can be considered as a likelihood of the DOA estimation result. In the proposed method, if $\left|\Delta \tau_{k}\right|$ exceeds a certain threshold, the estimated DOA is regarded as inaccurate value and it is replaced by the last DOA which does not exceed the threshold.

Considering the real environment, the received signal consists of To separate these, we use the band pass filter to identify each beacon.

\section{(iii) Prediction of the DOA from estimated location and pose}

The DOA is predicted based on estimated location and pose to be compare to the measured DOA and feedback the error in later step. Prediction is conducted by the following equation. Given $n$ sound sources with known locations, each sound source location is represented by $x_{k}$, $y_{k}$, where $k$ is the sound source number. The relationship between $x_{k}, y_{k}, \theta_{k}$ and the location $x, y$ and pose $\theta$ of the robot is expressed by,

$$
\left[\begin{array}{c}
\hat{\theta}_{1} \\
\hat{\theta}_{2} \\
\vdots \\
\hat{\theta}_{n}
\end{array}\right]=\left[\begin{array}{c}
\tan ^{-1}\left(\left(y_{1}-y\right) /\left(x_{1}-x\right)\right)-\theta \\
\tan ^{-1}\left(\left(y_{2}-y\right) /\left(x_{2}-x\right)\right)-\theta \\
\vdots \\
\tan ^{-1}\left(\left(y_{n}-y\right) /\left(x_{n}-x\right)\right)-\theta
\end{array}\right] .
$$

With this equation, the DOA is predicted from estimated location and pose and known sound source locations.

\section{(iv)Self-localization using odometry and DOA}

Location and pose of the robot which are estimated by odometry and estimated DOA are integrated by Extended Kalman filter. The proposed method utilize extended Kalman filter for self-localization by regarding equation (1) as state transition equation and equation (10) as observation equation. With these equations, the state of the robot $\boldsymbol{x}$ is estimated. We describe the detail of the extended Kalman filter in the following.

Let us define: $\hat{\boldsymbol{x}}_{t-\Delta t / t}$ as $\boldsymbol{x}$ on the time $t$ which is estimated on the time $t-\Delta t ; \hat{\boldsymbol{x}}_{t / t}$ and $\hat{\boldsymbol{x}}_{t / t+\Delta t}$ as $\boldsymbol{x}$ on the time $t$ and $t+\Delta t$ which is estimated on the time $t$; $\boldsymbol{P}_{t-\Delta t / t}, \boldsymbol{P}_{t / t}$ and $\boldsymbol{P}_{t / t+\Delta t}$ the covariance matrix of the estimation error of these estimates, respectively. The vector 
consists of the DOA estimated by (ii) is represented as $\boldsymbol{y}=\left[\theta_{1}, \theta_{2}, \ldots, \theta_{n}\right]^{\mathrm{T}}$ and the right member of the equation (10) is represented as $\boldsymbol{h}(\boldsymbol{x})$. Self-localization of the robot using extended Kalman filter is formulated as,

$$
\begin{aligned}
\boldsymbol{K} & =\boldsymbol{P}_{t-\Delta t / t} \boldsymbol{H}^{\mathrm{T}}\left[\boldsymbol{H} \boldsymbol{P}_{t-\Delta t / t} \boldsymbol{H}^{\mathrm{T}}+\boldsymbol{R}\right]^{-1}, \\
\hat{\boldsymbol{x}}_{t / t} & =\hat{\boldsymbol{x}}_{t-\Delta t / t}+\boldsymbol{K}\left(\boldsymbol{y}-\boldsymbol{h}\left(\hat{\boldsymbol{x}}_{t-\Delta t / t}\right)\right), \\
\boldsymbol{P}_{t / t} & =\boldsymbol{P}_{t-\Delta t / t}-\boldsymbol{K} \boldsymbol{H} \boldsymbol{P}_{t-\Delta t / t}, \\
\hat{\boldsymbol{x}}_{t / t+\Delta t} & =\boldsymbol{f}\left(\hat{\boldsymbol{x}}_{t / t}\right), \\
\boldsymbol{P}_{t / t+\Delta t} & =\boldsymbol{F} \boldsymbol{P}_{t / t} \boldsymbol{F}^{\mathrm{T}}+\boldsymbol{Q} .
\end{aligned}
$$

Here $\boldsymbol{R}$ is the covariance matrix of the observation error, which is the error of the DOA estimation, and $Q$ is the covariance matrix of the system noise, which is the error of the location and pose of the robot. $\boldsymbol{F}$ and $\boldsymbol{H}$ are the Jacobians which are defined as,

$$
\boldsymbol{F}=\left(\frac{\partial \boldsymbol{f}}{\partial \boldsymbol{x}}\right)_{\boldsymbol{x}=\hat{\boldsymbol{x}}_{t / t}}, \boldsymbol{H}=\left(\frac{\partial \boldsymbol{h}}{\partial \boldsymbol{x}}\right)_{\boldsymbol{x}=\hat{\boldsymbol{x}}_{t / t}} .
$$

By stepping $t+\Delta t \rightarrow t, t \rightarrow t-\Delta t$, the estimated self-location $\hat{\boldsymbol{x}}_{t / t}$ would be calculated recursively.

\section{Conditions of experiment Common condition}

To examine the ability of the proposed method, three types of experiments were conducted. At first, we note the common conditions of these. In the experiments, we have supposed the house-cleaning robot and its trajectory. The sound sources layout and the trajectory of the robot are shown in Fig. 5. The experiments were executed for 10

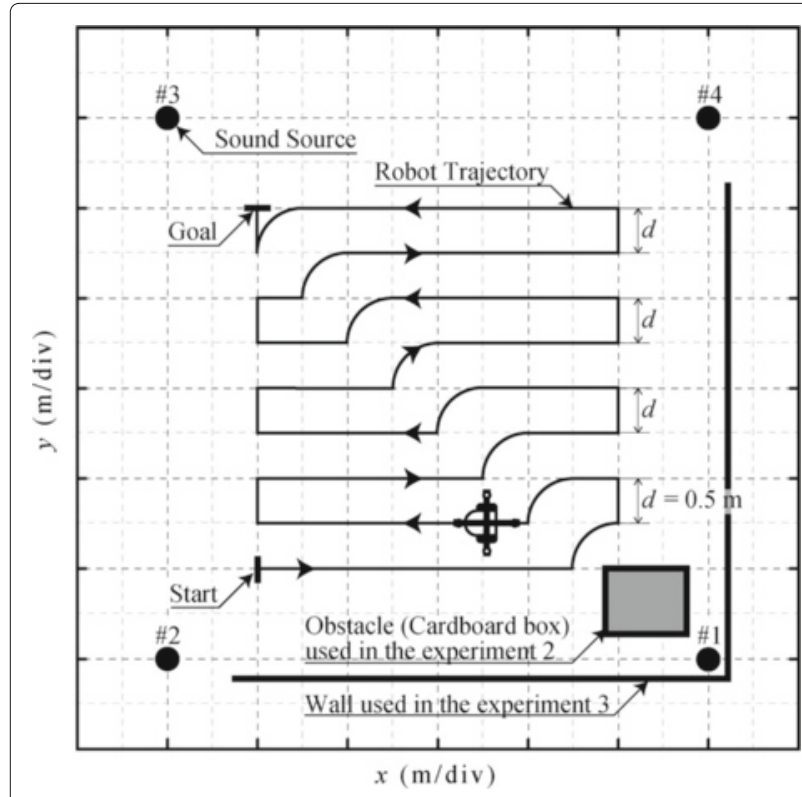

Fig. 5 Sound sources layout and the trajectory of the robot for experiment

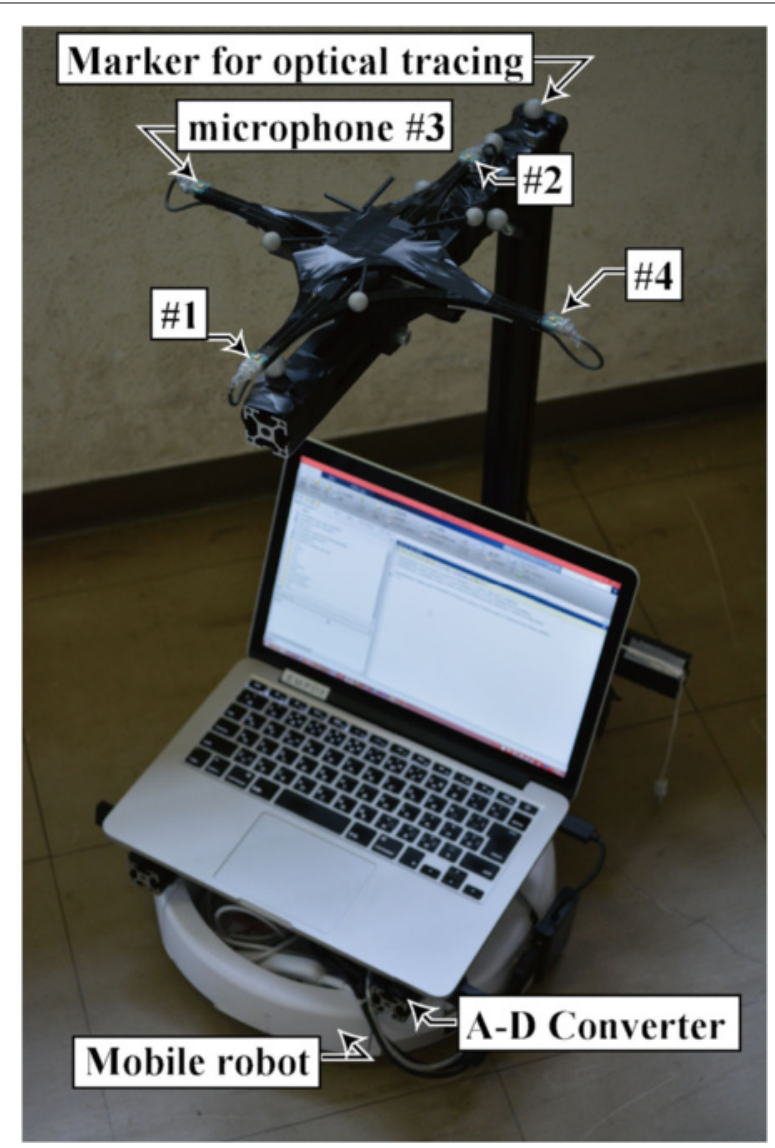

Fig. 6 Robot used for experiment. The marker was used to obtain ground truth using optical tracking

times respectively. The sampling frequency of the velocity and angular velocity, which were obtained by wheel rotation, was $5 \mathrm{~Hz}$.

The picture of the robot used in the experiment is shown in Fig. 6. On the robot, 4 elements of microphone array were placed, and the signals of them were acquired to personal computer using A-D converter. The sounds are recorded by MEMS microphone (SPU0414HR5H-SB, Knowles), which are the elements of the microphone array. The A-D converter was NI USB-6212 (National Instruments) and the sampling frequency of the A-D converter was $100 \mathrm{kHz}$. iRobot Create (iRobot Corporation) was used in the experiment. The velocity $v$ and angular velocity $\omega$ was obtained at $5 \mathrm{~Hz}$ using the Open Interface of the iRobot Create. These $v, \omega$ and the signals from microphone array are used for self-localization of each method.

The distances of the elements of the microphone array $d_{12}, d_{34}$ were both set to be $250 \mathrm{~mm}$. The sampling frequency of the microphone array was $100 \mathrm{kHz}$. 4 beacon sounds were placed on the 4 corners of the experimental environment. The band pass filters for sound sources 
separation were designed to have low-frequency cutoff at $f_{\mathrm{L}} \times 0.99(\mathrm{~Hz})$ and $f_{\mathrm{H}} \times 1.01(\mathrm{~Hz})$ for given sound with frequency band $\left[f_{\mathrm{L}} f_{\mathrm{H}}\right](\mathrm{Hz})$. The filters were implemented as finite impulse response filter which has tap number of 200. Other conditions are described in Table 1. The frequencies of the sound sources were chosen by considering frequency band of background noise and sharpness of autocorrelation of signal. The level of the sound sources were adjusted to maximum volume to achieve enough signal-to-noise ratio.

The window length $w$ was set to be $0.12 \mathrm{~s}$. The threshold of the value $\left|\Delta \tau_{k}\right|$ was set to be 0.2 , and if it exceeds the threshold, the DOA estimation is regarded as an inaccurate estimation. $\boldsymbol{R}, \boldsymbol{Q}$ used in the extended Kalman filter were set as,

$$
\begin{aligned}
& \boldsymbol{R}=1 \times 10^{6}\left[\begin{array}{cccc}
\Delta \tau_{1} & 0 & 0 & 0 \\
0 & \Delta \tau_{2} & 0 & 0 \\
0 & 0 & \Delta \tau_{3} & 0 \\
0 & 0 & 0 & \Delta \tau_{4}
\end{array}\right]+5 \boldsymbol{I}, \\
& \boldsymbol{Q}=\left[\begin{array}{ccc}
2 & 0 & 0 \\
0 & 2 & 0 \\
0 & 0 & 10
\end{array}\right] .
\end{aligned}
$$

Here $\Delta \tau_{1}, \Delta \tau_{2}, \Delta \tau_{3}$ and $\Delta \tau_{4}$ are the values of equation (9) for each sound source. Each of the constants are decided preexperimentally.

The ground truth of the location and pose of the robot need to be measured for evaluating the self-localization methods. In this experiment, the measurement of the ground truth was achieved by motion capture system with 18 cameras (OptiTrack Prime 41, OptiTrack) and analysis software (Motive body, OptiTrack). The frame rate of the system was set to 120 frames per second. The robot was equipped with 8 markers on the top of it.

Table 1 Condition of experiments

\begin{tabular}{lll}
\hline Robot velocity & 0.25 & $\mathrm{~m} / \mathrm{s}$ \\
$\begin{array}{l}\text { Sampling frequency of wheel rotation } \\
\text { Sampling frequency of microphones }\end{array}$ & 5 & $\mathrm{~Hz}$ \\
$\begin{array}{l}\text { Sound characteristics } \\
\text { Sound type }\end{array}$ & 100 & $\mathrm{kHz}$ \\
Sweeping time & 0.1 & Linear up chirp \\
Sweeping frequencies & & $\mathrm{s}$ \\
$\quad \begin{array}{l}\text { Sound 1 } \\
\text { Sound 2 }\end{array}$ & $12-14$ & $(\mathrm{kHz})$ \\
$\quad$ Sound 3 & $14.5-16.5$ & $(\mathrm{kHz})$ \\
$\quad$ Sound 4 & $17-19$ & $(\mathrm{kHz})$ \\
& $19.5-21.5$ & $(\mathrm{kHz})$ \\
\hline
\end{tabular}

\section{Conditions of specific experiments}

Conditions of experiment 1: without occlusion or reflective wave The robot runs on the trajectory without any obstacles or walls. This experiment was conducted to evaluate the localization error without these disturbances.

Conditions of experiment 2: occlusion of the sound source This experiment was conducted to evaluate the effect of the occlusion of sound source on the localization accuracy. Cardboard box (approximate dimensions height: $1 \mathrm{~m}$, width:0.5m for each) was placed as shown in Fig. 5 and it completely occlude sound source 1.

\section{Conditions of experiment 3: reflective wave from wall} This experiment was conducted to evaluate the effect of reflective wave on the localization accuracy. Wall was placed as shown in Fig. 5.

\section{Comparative methods}

Wheel-based odometry We compared the proposed method to the odometry using only wheel rotation, which is one of the conventional self-localization method and also a part of the proposed method. This method estimates self-location by updating $\boldsymbol{x}$ with equation (1) for every measurement. This is the odometry using only wheel rotation. As is clear from the equation (1), the measurement errors of $v$ and $\omega$ are not considered in this method although this method accumulates them. Because of this reason, this method has disadvantage that if there are the measurement errors on $v$ and $\omega$, they are accumulated over time.

Self-localization using only DOA estimation If location and DOA of each sound source are known, the location and pose of the robot can be estimated from them using equation (10). Let us define the difference of both members of the equation (10) as a function $\boldsymbol{h}_{s}(\boldsymbol{\theta}, \boldsymbol{x})$, where $\boldsymbol{\theta} \equiv\left[\theta_{1} \theta_{2} \ldots \theta_{n}\right]^{\mathrm{T}}$. If given DOA and location and pose of the mobile robot are consistent, $\boldsymbol{h}_{s}(\boldsymbol{\theta}, \boldsymbol{x})$ takes the value $\boldsymbol{0}$. Hereby, with given $\boldsymbol{\theta}$, self-localization by only DOA is achieved by,

$$
\hat{\boldsymbol{x}}=\min _{\boldsymbol{x}} \boldsymbol{h}_{s}(\boldsymbol{\theta}, \boldsymbol{x}) .
$$

When conducting (18), all DOA are assumed to be correct in this method. As mentioned before, the DOA is not always accurate as it is influenced by several disturbances such as reflective waves. Because of these reasons, the estimated location and pose of the robot is affected by the error of DOA.

\section{Results and discussion}

Experiment 1: without occlusion or reflective wave

Figures 7a shows an example of the self-localization results by each method for experiment 1 . As we have 

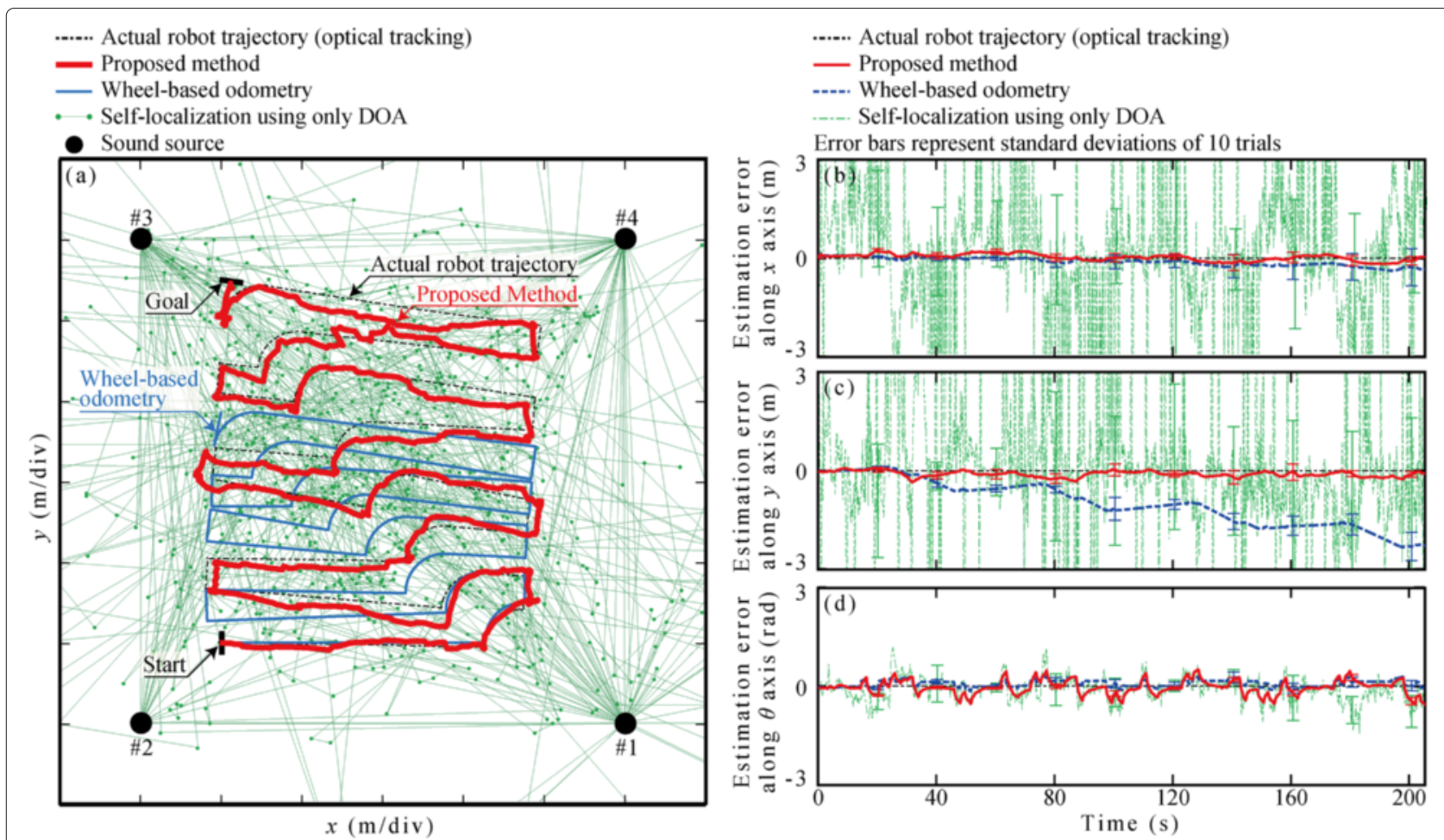

Fig. 7 Result of experiment 1: a An example of self-localization result in experiment $1 \mathbf{b}$ time variation of self-localization error along $x \mathbf{c}$ time variation of self-localization error along $y \mathbf{d}$ time variation of self-localization error along $\theta$

moved the robot open loop control, the real trajectory obtained by the optical tracking is slightly different from that of Fig. 5. Figures $7 \mathrm{~b}-\mathrm{d}$ shows the relation between time and self-localization errors along each axis for this experiment. The movie of the experiment and estimation are shown in Additional File 1.

From Fig. 7a, we can confirm that the proposed method estimates the real trajectory. Wheel-based odometry failed to estimate self-location as the distance between estimation result and actual trajectory was spread over time. However, sometimes the estimation result of the proposed method was incorrect when the odometry also have incorrect estimation. The proposed method combines the odometry and DOA and influenced by it.

From Fig. 7b-d, we can confirm that wheel-based odometry have estimation errors which increase over time. It indicates that measured velocity and angular velocity contains certain amount of errors and these are accumulated over time as mentioned before. By contrast, the proposed method does not have the errors which increase with time and achieve the estimation around the actual values. The standard deviation of them are almost equal to that of odometry and relatively smaller than that of the self-localizaiton using only DOA.

Figure 8 shows the DOA estimation error and $\Delta \tau_{k}$ for each sound sources over time. As we have defined before,

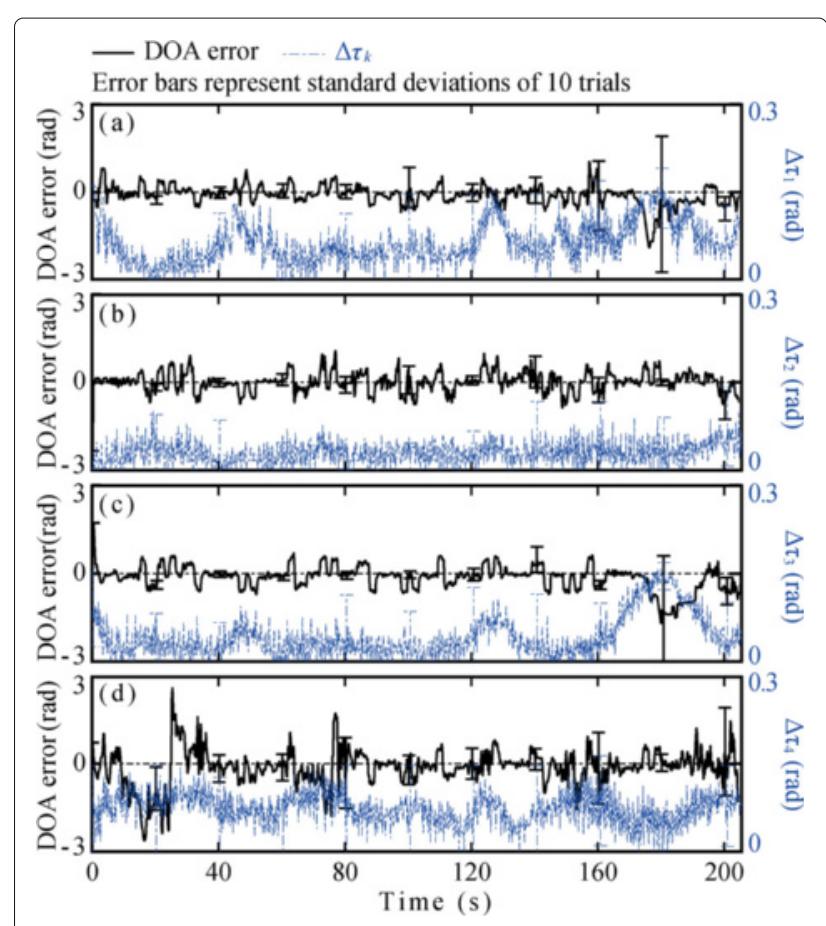

Fig. 8 DOA error and $\Delta \tau_{k}$ for each sound sources on experiment 1: a sound source $1 \mathbf{b}$ sound source 2 c sound source $3 \mathbf{d}$ sound source 4 
Table 2 Localization error of experiment 1 ( lower is better )

\begin{tabular}{lllll}
\hline Method & Error direction & Mean & Standard deviation & Unit \\
\hline Wheel-based odometry & Location $(x$ axis, $y$ axis) & $(-0.039,-0.672)$ & $(0.22,1.54)$ & 0.24 \\
& Pose & 0.077 & $(\mathrm{~m})$ \\
Self-localization using only DOA & Location $(x$ axis, $y$ axis) & $(2870,-1920)$ & 0.48 & $(\mathrm{rad})$ \\
& Pose & -0.069 & $(\mathrm{~m})$ & $(\mathrm{rad})$ \\
Proposed Method & Location $(x$ axis, $y$ axis) & $(0.040,-0.069)$ & $0.20)$ & $(\mathrm{m})$ \\
& Pose & -0.022 & $(\mathrm{rad})$ \\
\hline
\end{tabular}

$\Delta \tau_{k}$ shows the likelihood of the DOA estimation. If the $\Delta \tau_{k}$ was large, the DOA estimation result can be considered incorrect. $\Delta \tau_{k}$ were used in $R$, which was shown in the equation (17) and represents the variation of observation. If $\Delta \tau_{k}$ becomes large, the corresponding element of $R$ also becomes large and it will prevent the feedback of incorrect DOA from sound source $k$. By using these values, the estimation results can be stable even if the DOA error is huge. For example, the effect of it can be confirmed from Fig. 8d. Although the DOA estimation error was huge from $0 \mathrm{~s}$ to $40 \mathrm{~s}$ for sound source 4 and Self-localization using only DOA could not estimate the correct, the error does not affect to the localization result of the proposed method as shown in Fig. $7 \mathrm{~b}-\mathrm{d}$. The other example can be found in Fig. 8a and c. From
$160 \mathrm{~s}$ to $200 \mathrm{~s}$, the variation of the DOA error was relatively large. However, the large variation did not affect to the estimation result as the value of $\Delta \tau_{k}$ was also large.

Table 2 shows the mean and standard deviation of estimation errors of the location $x, y$ and the pose for each methods. From the experimental results, proposed method estimated the self-location with lower drift and variation of the estimation.

\section{Experiment 2: occlusion of the sound source}

Figure 9a shows an example of the self-localization results by each method for experiment 2 . Figure $9 b-d$ shows the relation between time and self-localization errors along each axis for this experiment.

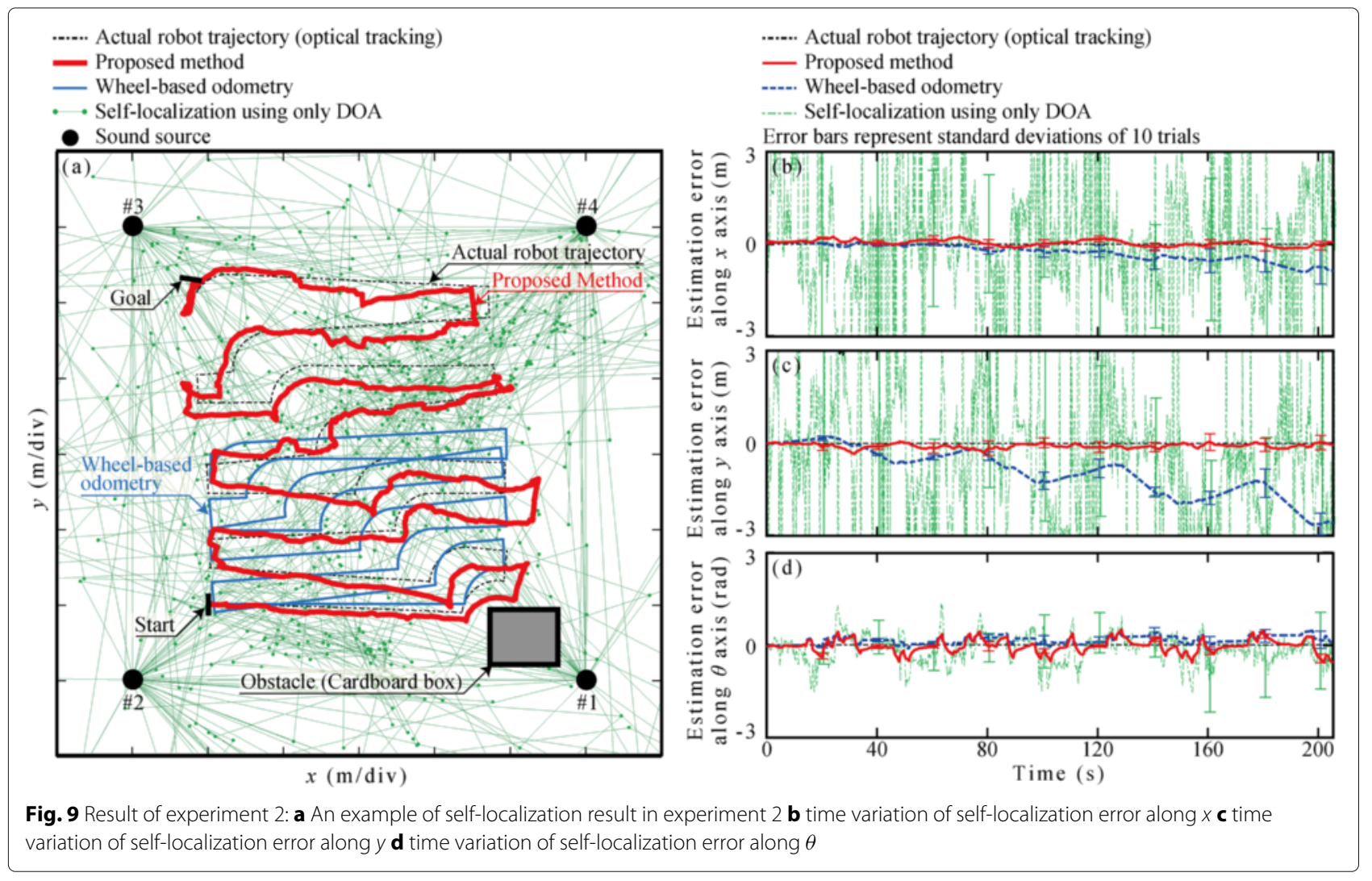




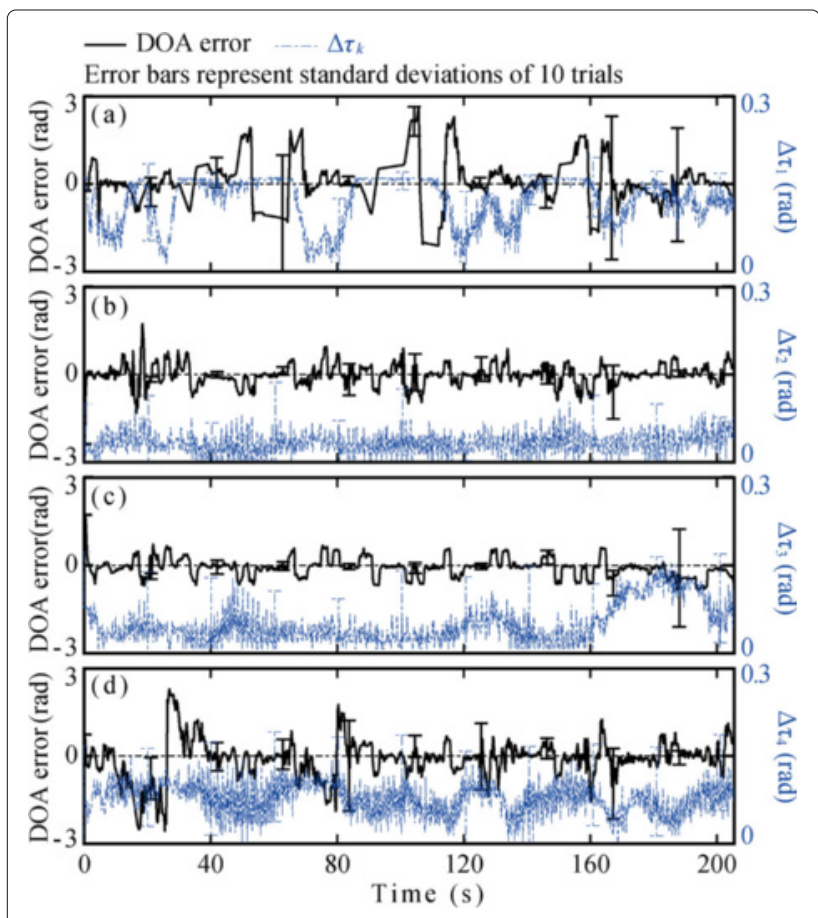

Fig. $10 \mathrm{DOA}$ error and $\Delta \tau_{k}$ for each sound sources on experiment 2: a sound source $1 \mathbf{b}$ sound source $2 \mathbf{c}$ sound source $3 \mathbf{d}$ sound source 4

From Fig. 9a, we can confirm that the localization result by proposed method was similar to that of optical tracking. However, it was relatively inaccurate than that of Fig. 7a.

Figure 10 shows the DOA estimation error and $\Delta \tau_{k}$ for each sound sources over time. The effect of the occlusion of sound source 1 can be considered in Fig. 10a. By comparing to the Fig. 8a, the DOA estimation result in experiment 2 have much error for most of the time. However, sometimes the DOA estimation was correct even the sound source 1 was occluded. In this case, $\Delta \tau_{k}$ of sound source 1 indicates that the DOA of it is not accurate, and as we can see in Figures $9 \mathrm{~b}-\mathrm{d}$, the DOA error did not have much effect to the localization result.

The reason of these can be considered as the diffracted wave from the sound source. With the diffracted wave, the microphones will receive multiple waves at once. It makes that estimated $\tau_{12, k}$ and $\tau_{34, k}$ from them would conflict and the conflict was detected by $\Delta \tau_{k}$. When $\Delta \tau_{k}$ was high, the proposed method suppress the feedback of sound source $\mathrm{k}$. In this case, it prevented the inaccurate DOA, which was affected by obstacle to be feedbacked.

Table 3 shows the mean and standard deviation of estimation errors of the location $x, y$ and the pose for each methods. The estimation errors are similar to that of experiment 1 , and localization error increased for approximately $60 \mathrm{~mm}$. From this result, we can confirm that the occlusion does not affect to the localization accuracy much if the other sound sources are not occluded.

\section{Experiment 3: reflective wave from wall}

Figure 11a shows an example of the self-localization results by each method for experiment 3 . Overall, proposed method shows similar estimation result compared to the previous 2 experiments in this example.

Figures $11 \mathrm{~b}-\mathrm{d}$ shows the relation between time and selflocalization errors along each axis for this experiment. As we have moved the robot open loop control, the robot runs into wall 2 times and we have removed these results and conducted analysis with 8 trials. The estimation error of the proposed method was high at the end of the estimation. The reason of this error can be considered as reflective wave from the wall. As shown in Fig. 12, the DOA estimation error at that time was relatively high for sound source 3 . Sound source 3 was facing to the center of field and its frequency was relatively high, When the robot was at the side of the sound source 3, the reflective wave from the wall could be larger than the direct wave because of its directivity. If the reflective wave was dominant in the microphone signal, the peak of the correlation function between microphones would exist at the time which represents time difference of reflective wave. In this condition, the reflective wave can be regarded as a sound source, and $\tau_{12, k}$ and $\tau_{34, k}$ did not conflict as much as that of diffracted wave in experiment 2 so that the value of the $\Delta \tau_{k}$ was not high. This problem can be solved by using omni-directional loudspeaker for the sound sources.

Table 4 shows the mean and standard deviation of estimation errors of the location $x, y$ and the pose for each

Table 3 Localization error of experiment 2( lower is better )

\begin{tabular}{lllll}
\hline Method & Error direction & Mean & Standard deviation & Unit \\
\hline Wheel-based odometry & Location $(x$ axis, $y$ axis) & $(-0.098,-0.590)$ & $(0.32,1.56)$ & 0.28 \\
& Pose & 0.154 & $(\mathrm{~m})$ \\
Self-localization using only DOA & Location $(x$ axis, $y$ axis) & $(1660,-2360)$ & 0.65 & $(\mathrm{rad})$ \\
& Pose & -0.046 & $(0.18,0.23)$ & $(\mathrm{m})$ \\
Proposed Method & Location $(x$ axis, $y$ axis) & $(0.045,-0.088)$ & 0.26 & $(\mathrm{~m})$ \\
& Pose & -0.029 & $(\mathrm{rad})$ \\
\hline
\end{tabular}




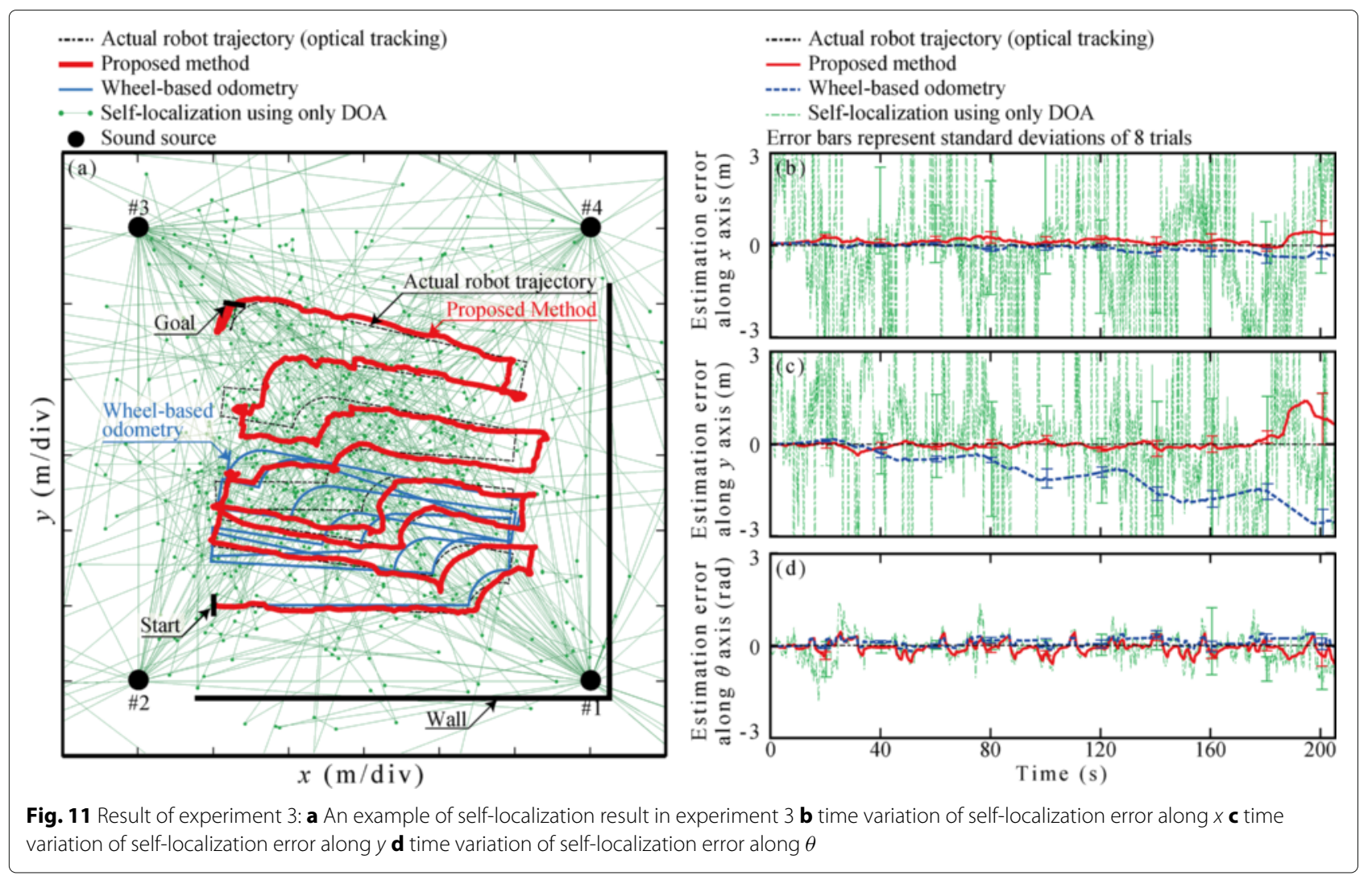

methods. The estimation error of the proposed method was relatively higher than that of experiment 1 , however, it is still acceptable for house-cleaning robot.

From these results, we can confirm that even with the obstacles or the walls, the proposed method can provide estimation result.

\section{Conclusion}

In this paper, we have proposed the low-cost selflocalization method which uses 4 elements of microphones, wheel rotation and known sound sources as beacons. The proposed method consists of following 4 steps. The proposed method (i) execute wheel-based odometry, (ii) estimate DOA of the sound sources using sounds recorded by the elements of the microphone array, (iii) predict the DOA of the sound sources from estimated location and pose, and (iv) conduct self-localization by integrating all of the information. To evaluate the proposed method, experiments were conducted. The proposed method was compared with the conventional methods, which were wheel-based odometry and selflocalization using only DOA. Three types of experiments were conducted to evaluate the proposed method with the occlusion or reflection of the sound. In experiment, the robot run on the trajectory which was supposed

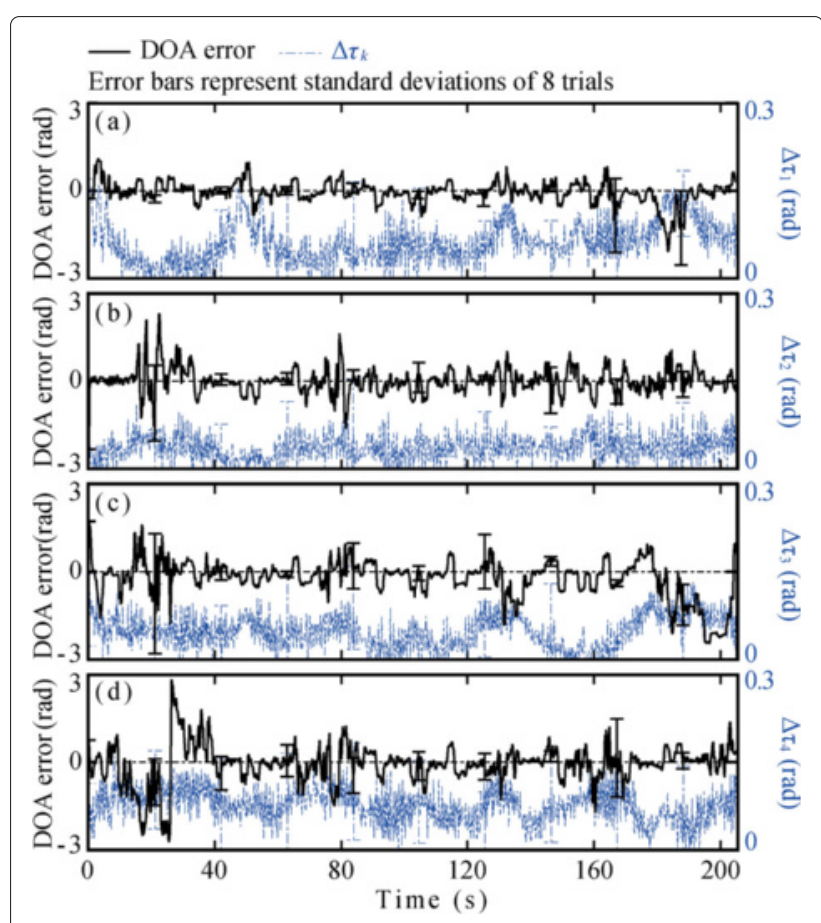

Fig. 12 DOA error and $\Delta \tau_{k}$ for each sound sources on experiment 3: a sound source $1 \mathbf{b}$ sound source $2 \mathbf{c}$ sound source $3 \mathbf{d}$ sound source 4 
Table 4 Localization error of experiment 3( lower is better )

\begin{tabular}{lllll}
\hline Method & Error direction & Mean & Standard deviation & Unit \\
\hline Wheel-based odometry & Location $(x$ axis, $y$ axis $)$ & $(-0.020,-0.49)$ & $(0.128,1.69)$ & 0.24 \\
& Pose & 0.096 & $(1.17,470)$ & $(\mathrm{m})$ \\
Self-localization using only DOA & Location $(x$ axis, $y$ axis) & $(620,-530)$ & 0.62 & $(\mathrm{~m})$ \\
& Pose & -0.076 & $(0.23,0.20)$ & $(\mathrm{rad})$ \\
Proposed Method & Location $(x$ axis, $y$ axis) & $(0.117,-0.047)$ & 0.26 & $(\mathrm{~m})$ \\
& Pose & -0.069 & $(\mathrm{rad})$ \\
\hline
\end{tabular}

the house cleaning robot. The experiments were conducted for 10 trials. As results, without any obstacles or walls, the mean of the estimation errors by wheelbased odometry were $670 \mathrm{~mm}$ and $0.08 \mathrm{rad}$, and those of self-localization using only DOA were $2870 \mathrm{~m}$ and $0.07 \mathrm{rad}$ in the worst case. In contrast with these methods, proposed method results in $69 \mathrm{~mm}, 0.02 \mathrm{rad}$ as the worst estimation error of self location and pose. Under the condition with occlusion, it affected to the DOA estimation of occluded sound source and proposed method detected the incorrect DOA. The increase of self-localization error by occlusion was approximately $60 \mathrm{~mm}$ in this condition. Under the condition with reflective wave, the localization error of the proposed method increased because of the directivity of sound source and reflective wave. It need to be clarified whether the omnidirectional speaker can solve this problem. From the results, the proposed method is enough feasible for indoor self-localization.

As future works, the effect of sound sources layout on the estimation accuracy and the effect of the multi-path on DOA estimation error need to be considered.

\section{Additional file}

Additional file 1: Overview of experiment and estimated results. In this movie, one trial of experiment and corresponding estimated results are shown. (MP4 $12.9 \mathrm{~kb})$

\section{Abbreviations \\ SLAM: Simultaneous localization and mapping; DOA: Direction-of-arrival.}

\section{Competing interests}

The authors declare that they have no competing interests.

\section{Authors' contributions}

All authors equally contributed. All authors read and approved the final paper.

\footnotetext{
Author details

${ }^{1}$ School of Integrative and Global Majors, University of Tsukuba, Ibaraki, JP.

${ }^{2}$ Graduate School of Systems and Information Engineering, University of

Tsukuba, Ibaraki, JP. ${ }^{3}$ Faculty of Engineering, Information and Systems,

University of Tsukuba, Ibaraki, JP.
}

Received: 9 April 2015 Accepted: 13 August 2015

Published online: 02 September 2015

\section{References}

1. Borenstein J, Feng L (1996) Gyrodometry: a new method for combining data from gyros and odometry in mobile robots. In: International Conference on Robotics and Automation, Minneapolis Vol. 1. pp 423-428. doi:10.1109/ROBOT.1996.503813

2. Lindstrom M, Eklundh $\mathrm{JO}$ (2001) Detecting and tracking moving objects from a mobile platform using a laser range scanner. In: 2001 IEEE/RSJ International Conference on Intelligent Robots and Systems (IROS). IEEE, Maui Vol. 3. pp 1364-1369. doi:10.1109/IROS.2001.977171

3. Murray D, Little JJ (2000) Using real-time stereo vision for mobile robot navigation. Auton Robot 8:161-171. doi:10.1023/A:1008987612352

4. Maeyama S, Ohya A, Yuta S (1995) Non-stop outdoor navigation of a mobile robot-retroactive positioning data fusion with a time consuming sensor system. In: Intelligent Robots and Systems 95. 'Human Robot Interaction and Cooperative Robots', Proceedings. 1995 IEEE/RSJ International Conference On Vol. 1. pp 130-1351. doi:10.1109/IROS.1995.525786

5. Aarabi P, Zaky S (2001) Robust sound localization using multi-source audiovisual information fusion. Inf Fusion 2(3):209-223. doi:10.1016/S1566-2535(01)00035-5

6. Thrun S, Leonard J (2008) Simultaneous localization and mapping. Springer handbook of robotics. In: Siciliano B, Khatib $O$ (eds). Springer handbook of robotics. Springer, Heidelberg. pp 871-889. doi:10.1007/978-3-540-30301-5_38

7. Thrun S, Fox D, Burgard W, Dellaert F (2001) Robust Monte Carlo localization for mobile robots. Artif Intell 128(1-2):99-141. doi:10.1016/S0004-3702(01)00069-8

8. Miura H, Yoshida T, Nakamura K, Nakadai K (2011) SLAM-based online calibration of asynchronous microphone array for robot audition. In: 2011 IEEE/RSJ International Conference on Intelligent Robots and Systems (IROS), San Francisco. pp 524-529. doi:10.1109/IROS.2011.6048869

9. Valin JM, Rouat J, Michaud F (2004) Enhanced robot audition based on microphone array source separation with post-filter. In: 2004 IEEE/RSJ International Conference on Intelligent Robots and Systems (IROS), Sendai Vol. 3. doi:10.1109/IROS.2004.1389723

10. Asono F, Asoh H, Matsui T (1999) Sound source localization and signal separation for office robot "JiJo-2". In: Proceedings. 1999 IEEE/SICE/RSJ. International Conference on Multisensor Fusion and Integration for Intelligent Systems. MFI'99. IEEE, Taipei. pp 243-248. doi:10.1109/MFI.1999.815997

11. Yamamoto S, Valin JM, Nakadai K, Rouat J, Michaud F, Ogata T, Okuno HG (2005) Enhanced robot speech recognition based on microphone array source separation and missing feature theory. In: Proceedings - IEEE International Conference on Robotics and Automation, Barcelona Vol. 2005. pp 1477-1482. doi:10.1109/ROBOT.2005.1570323

12. Saruwatari H, Mori Y, Takatani T, Ukai S, Shikano K, Hiekata T, Morita T (2005) Two-stage blind source separation based on ICA and binary masking for real-time robot audition system. In: 2005 IEEE/RSJ International Conference on Intelligent Robots and Systems (IROS), Edmonton. pp 209-214. doi:10.1109/IROS.2005.1544983

13. Nakadai $K$, Yamamoto $S$, Okuno HG, Nakajima H, Hasegawa Y, Tsujino H (2008) A robot referee for rock-paper-scissors sound games. In: Proceedings - IEEE International Conference on Robotics and Automation, California. pp 3469-3474. doi:10.1109/ROBOT.2008.4543741

14. Nakadai K, Nakajima H, Murase M, Okuno HG, Hasegawa Y, Tsujino H (2007) Real-Time Tracking of Multiple Sound Sources by Integration of 
Robot-Embedded and In-Room Microphone Arrays. J Robot Soc Japan 25(6):979-989. doi:10.7210/jrsj.25.979

15. Valin J.-m., Rouat J, Dominic L (2003) Robust Sound Source Localization Using a Microphone Array on a Mobile Robot. In: 2003 IEEE/RSJ International Conference on Intelligent Robots and Systems (IROS), Las Vegas. pp 1128-1233. doi:10.1109/IROS.2003.1248813

16. Silverman HF, Patterson WR, Flanagan JL (1997) The Huge Microphone Array (HMA). J Acoust Soc America 101(5):3119. doi:10.1121/1.418967

17. Weinstein E, Steele K, Agarwal A, Glass I (2004) Loud: A 1020-node modular microphone array and beamformer for intelligent computing spaces. Comput Sci Artif Intel Lab Tech Rep MIT-LCS-TM:1-18. http://129. 69.211.95/pdf/mit/lcs/tm/MIT-LCS-TM-642.pdf

18. Kawagishi T, Ogiso S, Mizutani K, Wakatsuki N (2014) Mobile Robot Localization using Sound Source Direction obtained by Small Element Number of Microphone Array. In: Proceedings of the 2014 JSME Conference on Robotics and Mechatronics. pp 2-208

19. Ogiso S, Kawagishi T, Mizutani K, Wakatsuki N, Zempo K (2015) Relation between sound sources layout and error of self-localization method in two-dimension for mobile robot using microphone array. In: Proceedings of the 22th International Congress on Sound \& Vibration (ICSV22), Florence. pp 01-010626

20. Zempo K, Mizutani K, Wakatsuki N (2013) Localization of Acoustic Reflective Boundary Using a Pair of Microphones and an Arbitrary Sound Source. Japan J Appl Phys 52(7S):07-06

21. Zempo K, Mizutani K, Wakatsuki N (2013) Suppression of Noise Using Small Element Number of Microphone Array in Reflective. In: Proceedings of the 20th International Congress on Sound \& Vibration (ICSV20), Bangkok. pp 05-649

22. Mizutani K, Ebihara T, Wakatsuki N, Mizutani K (2009) Locality of area coverage on digital acoustic communication in air using differential phase shift keying. Japan J Appl Phys 48(7 PART 2):07-07. doi:10.1143/JJAP.48.07GB07

\section{Submit your manuscript to a SpringerOpen ${ }^{\circ}$ journal and benefit from:}

- Convenient online submission

- Rigorous peer review

- Immediate publication on acceptance

- Open access: articles freely available online

- High visibility within the field

Retaining the copyright to your article

Submit your next manuscript at $\gg$ springeropen.com 